\title{
Surprise et saturation
}

\section{Claudia Serban}

\section{OpenEdition \\ Journals}

Édition électronique

URL : http://journals.openedition.org/alter/424

DOI : 10.4000/alter.424

ISSN : 2558-7927

\section{Éditeur :}

Association ALTER, Archives Husserl (CNRS-UMR 8547)

\section{Édition imprimée}

Date de publication : 1 décembre 2016

Pagination : 69-80

ISBN : 978-2-9550449-2-6

ISSN : 1249-8947

\section{Référence électronique}

Claudia Serban, « Surprise et saturation », Alter [En ligne], 24 | 2016, mis en ligne le 01 décembre 2017, consulté le 19 avril 2019. URL : http://journals.openedition.org/alter/424 ; DOI : 10.4000/alter.424 


\section{SURPRISE ET SATURATION}

\section{Claudia Serban}

$\mathrm{Au} \S 74$ de l'Anthropologie d'un point de vue pragmatique, Kant propose une définition remarquable de l'affect comme une "surprise par la sensation (Überraschung durch Empfindung) »1. La vie affective serait, dans cette perspective, une vie de surprises. Mais l'on peut sans doute aller encore plus loin et viser à montrer que la surprise ne recouvre et n'exprime pas seulement le surgissement de l'affect, mais aussi, plus fondamentalement, le mouvement de la phénoménalisation. En proposant dans ce qui suit une lecture des pages que JeanLuc Marion a consacrées, dans Réduction et donation et dans Étant donné, à la surprise, nous allons essayer de défendre la thèse suivante, qui représente un déplacement de la thèse kantienne : Le phénomène, en tant qu'il se donne - donc en tant que donné - est une surprise par la saturation.

En avançant cette thèse, nous mobilisons implicitement une compréhension de la surprise qui y voit beaucoup plus qu'une émotion parmi d'autres, et même plus qu'un trait commun à toutes les émotions - une passion primitive, comme l'admiration qui, au $\S 53$ des Passions de l'âme, apparaît à Descartes comme la "première de toutes les passions ». Notre hypothèse est en effet que la surprise décrit non seulement le surgissement de l'affect, mais le surgissement du phénomène. En ce sens, nous allons essayer de proposer, en nous appuyant sur les analyses de Jean-Luc Marion, une thèse proprement phénoménologique sur la surprise, en nous concentrant sur le rapport entre surprise et saturation.

Quel serait cependant l'intérêt de cette thèse ou, autrement dit, l'intérêt d'introduire la surprise en phénoménologie? Déjà, il faut

\footnotetext{
${ }^{1}$ Foucault traduit : «Dans l'émotion, l'esprit surpris par l'impression perd l'empire de soi-même (animus sui compos)» (I. Kant, Anthropologie du point de vue empirique, Paris, Vrin, 1984, p. 109).
} 
noter que la phénoménologie historique n'a pas été indifférente à la surprise, ou plus précisément: à l'expérience de la surprise. Pour se limiter à Husserl et à Heidegger et pour commencer avec ce dernier, il suffit de rappeler le $\S 69$ d'Etre et temps, portant sur la «Temporalité de l'être-au-monde et le problème de la transcendance du monde », où nous pouvons lire :

[...] la possibilité de l'être-surpris (Überraschtwerden) par quelque chose se fonde en ceci que le présentifier attentif (das gewärtigende Gegenwärtigen) d'un à-portée-de-la-main (Zuhandenes) est in-attentif (ungewärtig) à un autre à-portée-de-la-main se tenant avec le premier dans un contexte possible de tournure. C'est l'in-attention propre au présentifier perdu (Das Ungewärtigen des verlorenen Gegenwärtigens) qui ouvre pour la première fois l'espace de jeu «horizontal» à l'intérieur duquel du surprenant (Überraschendes) peut assaillir (überfallen) le Dasein².

Pour Heidegger, c'est donc l'inattention, c'est-à-dire une défaillance de la temporalité authentique et de la vigilance du Dasein, qui fonde la possibilité de la surprise. Chez Husserl, d'autre part, la place de la surprise dans l'expérience se définit dans l'horizon de l'opposition entre remplissement et déception ${ }^{3}$. Le sens de la surprise est, dans cette perspective, d'être une attente déçue, ou une intention non remplie ${ }^{4}$, pour autant qu'elle rompt le déroulement concordant de l'expérience et y introduit la discordance sous la forme du nonremplissement de la visée. Selon toute apparence donc, ni Husserl ni Heidegger n'arrivent à proposer une compréhension positive de la surprise, ou de saisir la surprise dans sa positivité. Bien plutôt, l'expérience de la surprise est pour eux la marque d'un manque, d'un défaut, d'une privation ${ }^{5}$.

Nous retrouvons une telle connotation négative de la surprise dans un autre contexte, chez Valéry, dans le cadre de la réflexion sur

\footnotetext{
${ }^{2}$ M. Heidegger, Sein und Zeit, Tübingen, Niemeyer, 2001 [1927], p. 355. Traduction Emmanuel Martineau, édition numérique hors commerce.

${ }^{3}$ Pour cette opposition, voir par exemple le $\S 9$ de la Quatrième Recherche logique (in E. Husserl, Recherches logiques II. Recherches pour la phénoménologie et la théorie de la connaissance, trad. H. Élie, A. L. Kelkel et R. Schérer, Paris, PUF, «Épiméthée », p. 108, note 1; Husserliana, XIX/1, Logische Untersuchungen, vol. II, première partie: Untersuchungen zur Phänomenologie und Theorie der Erkenntnis, Ursula Panzer (éd.), Dordrecht/Boston/Londres, Kluwer Academic Publishers, 1984, p. 323 , note).

${ }^{4}$ Voir en ce sens les analyses du $\S 21$ d'Expérience et jugement, qui situent la déception de l'intention sous le signe de l'empêchement et du conflit.

${ }_{5}^{5}$ Pour le traitement de la surprise dans la phénoménologie post-heideggérienne, nous renvoyons à l'article de Natalie Depraz: «Phenomenology of Surprise. Levinas and MerleauPonty in the Light of Hans Jonas ", in Advancing Phenomenology: Essays in Honor of Lester Embree, Dordrecht, Springer 2010, p. 223-233.
} 
l'époque contemporaine que propose la conférence de 1935 intitulée Le bilan de l'intelligence, où est déplorée l' «impression générale d'impuissance et d'incohérence » que provoquent « trop de surprises, trop de créations, trop de destructions », qui font que "tout le monde est comme surpris » et que "nous sommes, chaque jour, à la merci d'une invention $»^{6}$. La surprise apparaît ici comme le symptôme de l'incohérence de l'époque et du monde contemporain, bref comme un signe de la crise, comme si l'élimination de la surprise pouvait être le gage de la stabilité et de la solidité de notre monde et de notre existence. $\mathrm{Au}$ diagnostic négatif de Valéry nous pouvons cependant opposer celui de Jean-Luc Marion, qui déplore inversement, dans ses Entretiens avec Dan Arbib publiés sous le titre La rigueur des choses, le « pessimisme profond et terrifié par la surprise de l'instant suivant » $\mathrm{du} \ll$ nihilisme, qui assure que rien ne se passera $»^{7}$.

Toutes ces considérations indiquent déjà que les enjeux d'une réflexion sur la surprise, sur son statut et sa place dans notre expérience, ne sont pas insignifiants ou négligeables, et ce même avant d'adopter véritablement le fil conducteur que nous avons annoncé, celui d'une réflexion phénoménologique sur la surprise considérée dans son rapport à la saturation du phénomène et de l'expérience. En mobilisant les analyses et les résultats de la phénoménologie de la saturation élaborée par Jean-Luc Marion depuis Étant donné (1997), il s'agira donc de montrer tout d'abord que le prisme de la saturation permet de penser la surprise comme une expérience pleine et positive, et non plus seulement comme une privation.

\section{Analyses et descriptions de la surprise}

Mais si nous partons à ce dessein des textes eux-mêmes, nous serons confrontés au fait suivant : la surprise ne figure pas, dans Étant donné, parmi les déterminations du donné ( $\$ 13$ à 18), ni n’apparaît au moment où prend contour l'esquisse du phénomène saturé $(\S 21$ et 22). L'endroit où la surprise est explicitement mentionnée et thématisée est en effet le $\S 26$, au livre $\mathrm{V}^{8} \mathrm{~d}^{\prime} E ́ t a n t$ donné qui est consacré à ce

\footnotetext{
${ }^{6}$ P. Valéry, Le bilan de l'intelligence (1935), Paris, Allia, 2011, p. 17 et 18.

7 J.-L. Marion, La rigueur des choses. Entretiens avec Dan Arbib, Paris, Flammarion, 2012, p. 267 et 273.

8 J.-L. Marion, Étant donné. Essai d'une phénoménologie de la donation, Paris, PUF, "Épiméthée», 1997 (désormais noté ED). Ce n'est peut-être pas inutile de rappeler la structure de cet ouvrage : le premier livre - la donation - établit l'universalité de la donation; le livre II - le don - opère la reconduction du don à la donation; le livre III est consacré aux déterminations du donné : l'anamorphose, l'arrivage, le fait accompli, l'incident, l'événement, l'étant donné ; le livre IV,
} 
que Jean-Luc Marion appelle l'adonné et qui désigne, pour le dire brièvement, le destinataire ou l'attributaire du phénomène saturé, ou encore celui qui reçoit ce qui se donne.

La première détermination de l'adonné n'est cependant pas la surprise, mais l'appel. La surprise n'est qu'un des quatre caractères de l'appel, à côté de la convocation, de l'interlocution et de la facticité. Si l'on remonte toutefois au moment où cette centralité de l'appel se met en place, à savoir, à Réduction et donation (1989), force nous est de constater que la surprise se trouve inscrite d'entrée de jeu au cœur de l'appel: "L'appel même intervient comme tel, sans ou avant tout autre message que de "surprendre" celui qui l'entend, de prendre même celui qui ne l'attend pas $»^{9}$. C'est pourquoi le premier nom de l'adonné est bien l'interloqué, c'est-à-dire, celui qui est par définition surpris par l'appel. L'appel lui-même agit donc par surprise et en surprenant. Il est utile de rappeler ici ce passage éloquent:

L'interloqué subit une surprise. Il faut entendre ici la surprise moins comme un étonnement ou une stupeur, que comme l'antagoniste de toute extase - tant celle de l'intentionnalité que celle de la " résolution anticipatrice »; car, si l'extase institue sans doute le sujet hors de lui-même, elle ne l'extériorise jamais qu'à partir de lui seul et pour le disposer à revenir sur lui-même ; une telle extase confirme le sujet dans sa fonction de pôle originaire et la présuppose même dans l'excès du dépassement. Au contraire, la surprise s'empare de l'interloqué à partir d'un lieu et d'un événement absolument étranger, en sorte d'annuler toute prétention d'un sujet à constituer, reconstituer ou décider de ce qui le surprend ; la surprise prend l'interloqué, en ce qu'elle le déprend de toute subjecti(vi)té, s'en prend à toute polarité auto-constituante en lui, et finalement le comprend à partir et dans un événement que l'interloqué, lui, ne comprend en aucune manière (comprendre en effet suppose une prise de possession, que la simple connaissance $n^{\prime}$ impose pas). Littéralement, la surprise interdit à $l^{\prime}$ interloqué de comprendre la convocation qu'il reçoit pourtant ${ }^{10}$.

De cette description, nous pouvons dégager au moins deux éléments constitutifs de la surprise : d'une part, le fait qu'elle se distingue radicalement $d^{\prime}$ un acte par lequel le sujet se pose lui-même ou $\mathrm{s}^{\prime}$ institue comme maître et possesseur de ce qui lui advient. Bien au contraire, en tant qu'il subit la surprise de l'appel, l'interloqué est essentiellement «destitué d'autarcie ». Dans cette perspective, la sur-

aux degrés du donné et à l'esquisse du phénomène saturé ; et le livre $\mathrm{V}$, le dernier, à l'adonné, qui non seulement reçoit, mais se reçoit de ce qui se donne.

9 J.-L. Marion, Réduction et donation. Recherches sur Husserl, Heidegger et la phénoménologie, Paris, PUF, «Épiméthée »,1989 (désormais noté RD), p. 295.

${ }_{10}$ RD, p. 300-301. 
prise n'est pas seulement ce qui déborde toute prise du sujet, mais elle va jusqu'à inverser cette prise : au lieu de prendre (acte, conscience, etc.), le sujet est «pris par la surprise ${ }^{11}$. La posture de l'interloqué semble donc être, de prime abord, celle d'une totale et foncière passivité. Nous aurons à revenir sur ce point important. $\mathrm{D}^{\prime}$ autre part, et en tant que deuxième élément constitutif de la surprise, nous pouvons noter son caractère incompréhensible: la surprise est subie ou reçue sans être comprise, car la comprendre, ce serait déjà une manière de ne pas être surpris. Autrement dit : «La surprise surprend, précisément parce qu'elle surprend celui qui en ignore le nom et le vœu; je me découvre interloqué, précisément parce que je ne sais, dans l'instant de la revendication, ni qui, ni quoi ». Être véritablement surpris, c'est ne pas comprendre, ne pas savoir. Et pourtant, quelque chose advient ou est advenu, il y a ou il y a eu expérience : "Sans savoir ni par qui, ni par quoi, je me sais dès $l^{\prime}$ origine déjà interloqué $»^{12}$.

L'appel, la revendication, la convocation agissent donc "par surprise ${ }^{13}$. Cette conclusion est reprise et développée par Étant donné presque point par point : nous y retrouverons donc les éléments qui viennent d'être mis en évidence. Tout d'abord, l'inversion de la prise dans la sur-prise pourra être précisée, dans le sillage de Levinas, comme une inversion de l'intentionnalité, ou comme une "contreintentionnalité ${ }^{14}$. Cette inversion apparaît en même temps comme l'effet rigoureux de la saturation : "C'est donc le phénomène saturé comme tel qui inverse l'intentionnalité et soumet l'attributaire à la présence de l'appel ${ }^{15}$. Cette soumission de l'attributaire - qui, d'interloqué, devient adonné -se reflète dans le passage du nominatif à l'accusatif: on ne dit plus "Je pose», "Je constitue», mais " cela m'advient », " cela me surprend », « cela m'appelle ». Dans cet infléchissement s'exprime donc aussi l'inversion de la prise ou de l'emprise en surprise :

Surprise: l'interloqué, résultant d'une convocation, se reconnaît pris et surplombé (sur-pris) par une emprise. Mais cette emprise le détermine d'autant plus radicalement qu'elle reste (ou peut rester) d'origine indéterminée. L'appel surprend en prenant l'adonné sans pourtant toujours lui apprendre quoi que ce soit; il le réduit seulement aux aguets, le fige en arrêt, le met en disponibilité

\footnotetext{
11 Ibid., p. 301.

12 Ibid., p. 302.

${ }_{13}$ Ibid., p. 301.

14 ED, p. 367.

15 Ibid., p. 369.
} 
immobile pour ce qui, justement, peut ne pas en finir de venir, voire ne même pas commencer. Pareil écart, imposé au moi/me sans lui en donner connaissance, contredit donc toute extase de la connaissance, où le $J e$ transcendantal constituait, devant lui et en une évidence par principe transparente, l'objet. La surprise, cette emprise obscure et subie [nous soulignons], contredit l'intentionnalité, cette extase connue et connaissante déployée par le Je à partir de lui-même; loin de surveiller du regard le terrain clair de l'objectivité à connaître, le Je transmué en un moi/me se reconnaît surplombé par la revendication inconnaissable ${ }^{16}$.

Nous voyons se dégager ici avec force une caractérisation de la surprise comme "emprise obscure et subie », et nous retrouvons sous cette forme ses deux traits constitutifs déjà évoqués: la passivité qu'elle constitue, et son incompréhensibilité ou inconnaissabilité. Il semblerait donc que, le plus souvent (mais pas toujours), la surprise se fait sans apprentissage : elle advient sans nous apprendre quoi que ce soit. Le gain cognitif de la surprise paraît donc, au moins à première vue, incompatible avec le potentiel surprenant qui la définit. On ne saurait être instruit par la surprise, comme on est, selon Eschyle, « instruit par la souffrance (pathei mathos)». La surprise reste obscure, opaque en quelque sorte. Elle est aussi subie: elle nous arrive sans qu'on soit allé à son encontre. Devancer la surprise, l'anticiper, ce serait lui ôter son caractère même de surprise.

Nous pouvons cependant nous demander s'il ne faut pas être préparé d'une certaine façon à la surprise pour la recevoir en tant que telle, pour pouvoir être affecté par elle. L'impassibilité pourrait en effet être tout aussi fatale à la surprise que l'anticipation - ce que certaines pathologies comme la dépression et la mélancolie, qui altèrent et mettent en danger la possibilité même d'être surpris, prouvent éminemment. C'est pourquoi la «disponibilité immobile» de l'adonné ne doit pas être comprise comme une pure passivité, mais bien plutôt comme une forme de vigilance et $d^{\prime}$ attention ${ }^{17}$. On peut aussi se demander si la surprise est nécessairement ou entièrement obscure, si elle ne peut pas être aussi en un sens éclairante. Comprise comme vigilance, la disponibilité pour la surprise serait déjà une lumière dans l'obscurité, ou une forme de clarté, sans laquelle la surprise risquerait peut-être tout simplement de ne pas être subie, de ne pas entrer dans l'expérience. Mais avant de formuler d'autres questions, avançons dans la lecture du texte :

\footnotetext{
${ }^{16}$ Ibid., p. 370. Nous soulignons.

${ }_{17}$ Pour reprendre les concepts mis en avant par Natalie Depraz dans Attention et vigilance. À la croisée de la phénoménologie et des sciences cognitives, Paris, PUF, «Épiméthée », 2014.
} 
L'inversion du surplomb (sur-prise) ne fait donc qu'un avec la disqualification de la compréhension objectivante (sur-prise). L'une et l'autre se confondent dans la même perte de connaissance, au double sens d'une perte de toute conscience originelle de soi et d'une impuissance à saisir le pôle originel de la revendication comme un objet. Descartes peut servir ici de guide grâce à sa définition de l'admiration: "Lorsque la première rencontre de quelque objet nous surprend, et que nous le jugeons être nouveau, ou fort différent de ce que nous connaissions auparavant ou que nous supposions qu'il devait être, cela fait que nous l'admirons et que nous sommes étonnés. Et parce que cela peut arriver avant que nous connaissions aucunement si cet objet nous est convenable, ou s'il ne l'est pas, il semble que $l^{\prime}$ admiration est la première de toutes les passions $»^{18}$.

Ce n'est donc pas toute compréhension qui est mise hors jeu dans la surprise, mais seulement la compréhension objectivante. Subir une surprise, c'est cesser d'être confronté à un objet, à quelque chose qui se laisse déterminer et maîtriser comme un objet; mais c'est aussi cesser d'être un sujet maître de l'apparition, pour devenir au contraire sujet à l'apparition. Si la référence à Descartes qui intervient dans ce contexte indique la manière dont la première des passions de l'âme, l'admiration, est le résultat d'une rencontre par surprise, de la surprise de la nouveauté, surprise qui a lieu avant toute détermination de ce qui est rencontré comme bon ou mauvais, comme utile ou nuisible,

ce rapprochement a pourtant sa limite : pareille surprise de l'admiration n'en reste cependant pas moins, pour Descartes, une première passion de l'ego, donc du "sujet»; or nous décrivons ici une affection plus originaire, qui précède la subjecti(vi)té métaphysique ; même si, éventuellement, celle-ci pouvait encore en procéder, la surprise la destituerait de son privilège de fondement métaphysique, puisqu'elle met en œuvre l'a posteriori originaire du dernier principe $(\S 1)^{19}$.

La surprise est donc une affection plus originaire que l'admiration cartésienne, pour autant qu'elle n'est pas la passion d'un sujet déjà constitué et autarcique, mais ce qui, comme le disait déjà Réduction et donation, destitue le sujet de son autarcie, voire qui précède l'institution même du sujet et, ce faisant, le détermine comme interloqué ou adonné.

\footnotetext{
${ }^{18}$ ED, p. 370. La citation donnée est tirée du § 53 des Passions de l'âme.

${ }_{19} \mathrm{Ibid}$., p. 371. Le principe auquel il est fait référence est celui qu'énonçait déjà, dans sa conclusion, Réduction et donation, et que le $\S 1$ d'Étant donné reprend, développe et justifie : Autant de réduction, autant de donation.
} 


\section{La surprise ou l'événement?}

Nous avons examiné jusqu'à présent la manière dont la surprise intervient pour déterminer l'action de l'appel et l'institution de l'interloqué ou de l'adonné. Nous pouvons cependant poser une question plus générale à propos du rôle relativement minimal et marginal de la surprise dans l'économie interne d'une phénoménologie de la donation et de la saturation. Ne pourrait-on pas, en effet, élargir la portée descriptive de la surprise et y voir une détermination, non seulement de l'adonné, mais du phénomène saturé lui-même?

En formulant cette question, nous retrouvons notre hypothèse de départ, selon laquelle le phénomène, en tant qu'il se donne - donc en tant que donné - est une surprise par la saturation. Pour étayer cette hypothèse, il nous faudra montrer que le surcroît de la saturation, que thématise Jean-Luc Marion, se laisse expliciter précisément comme surprise. Ce faisant, nous voudrions montrer que la surprise pourrait s'avérer tout aussi importante que l'événement pour la description du phénomène saturé. Pourquoi introduire ici la considération de l'événement en rapport avec la surprise? Tout simplement parce qu'on peut avoir tendance à classer la surprise, lorsqu'on en fait un usage conceptuel plus élargi, sous le signe d'une "phénoménologie de l'événement $»^{20}$. En prenant soin de ne pas substituer la surprise à l'événement (car ils ne se situent pas, visiblement, sur le même plan), nous allons au contraire tenter d'émanciper la surprise de la tutelle de l'événement pour en faire une coordonnée du phénomène saturé à part entière.

Il faut partir à cette fin des déterminations du donné que l'on trouve au livre III d'Étant donné, et en particulier de quatre d'entre elles, à savoir l'arrivage (§ 14), l'incident (§ 16), le fait accompli (§ 15) et l'événement ( $\S 17)$, même si l'anamorphose, qui vient en première position, reflète peut-être déjà au mieux l'inversion de la prise du sujet métaphysique ou transcendantal en sur-prise. Mais plutôt que de contreintentionnalité, il s'agit dans ce contexte d'excès, ou de ce que nous pourrions appeler le surcroît de la surprise. C'est cet excès qui rompt la continuité de l'apparaître et crée la brèche où s'installe la saturation ou l'éblouissement : « Plutôt que d'arrivées, il faut donc parler d'arrivages de phénomènes, selon des rythmes discontinus, par saccades, inopinés, par surprise, détachés les uns des autres, par rafales, stochasti-

\footnotetext{
${ }^{20}$ Voir l'article de F. Dastur : « Phénoménologie de l'événement : l'attente et la surprise », paru en 1997 dans la revue Études phénoménologiques et repris dans le volume La phénoménologie en questions, Paris, Vrin, 2004.
} 
ques... $»^{21}$. La « contingence originelle du phénomène $»^{22}$, que désigne l'arrivage, s'accomplit donc comme et par surprise.

Mais plus loin encore, la surprise pourrait aussi exprimer la temporalité propre au phénomène. Si le terme français ne comporte pas de connotation temporelle immédiate, le mot allemand (Überraschung) contient directement une détermination temporelle : celle de l'instantanéité ou de la fulgurance, qui résonne de près avec l'exaiphnès platonicien (Lettre VII, 341c). Le modèle temporel ainsi introduit est donc celui de la rupture ou de l'interruption, mais non pas nécessairement au sens d'un "phénomène sans généalogie » ${ }^{23}$. Bien plutôt, si la surprise, comme l'événement "précède sa cause (ou ses causes) » et n'est susceptible donc que d'une explication rétrospective, c'est dans la mesure où ce n'est pas nous qui la devançons (par une quelconque "résolution anticipatrice »), mais c'est elle qui nous devance en se devançant elle-même (on pourrait donc parler à ce propos, pour employer un terme de Natalie Depraz, d'auto-antécédence ${ }^{24}$ ).

Nous pouvons donc ressaisir l'arrivage du phénomène considéré en tant que donné et sa temporalité propre à partir de la surprise. Mais il faut aller plus loin et montrer que la surprise exprime l'excédent même du donné, ou le surcroît de la saturation. L'excédent est en effet la marque du phénomène saturé, par contraste avec la pauvreté du phénomène commun - de ce phénomène dont nous dirons qu'il ne nous surprend pas ou ne nous interpelle pas. D'une part, en effet, «tout phénomène, en tant que donné, garde en effet comme un surplomb sur ce qui le reçoit $»^{25}$. Nous avons vu plus haut que ce surplomb du phénomène s'exerçant sur l'ego déplacé du nominatif à l'accusatif désigne l'action même de la surprise. Mais d'autre part: «Plus l'excès se constate, plus l'événement s'impose ${ }^{26}$. L'événement et la surprise croissent donc proportionnellement, et l'on pourrait même aller jusqu'à dire que l'excès de l'événement s'identifie au surcroît de la surprise. En effet: «L'événement, je peux l'attendre (quoique le plus souvent il me surprenne) $»^{27}$. Tout autant qu'irregardable (pour reprendre la détermination négative proposée par le $\$ 22$ d'Étant donné), l'événement est par définition surprenant. S'il advient

\footnotetext{
${ }^{21}$ ED, p. 186.

22 Ibid., p. 187.

${ }^{23}$ Ibid., p. 199.

24 N. Depraz, Lucidité du corps: De l'empirisme transcendantal en phénoménologie, Dordrecht, Springer, 2001, partie II, par ex. p. 79 et 104.

${ }_{25}$ ED, p. 224.

26 Ibid., p. 241.

27 Ibid., p. 226.
} 
véritablement comme un événement, c'est en débordant la prise que l'on croit avoir sur lui. S'il ne nous surprend pas, c'est que nous l'avons prévu et compris d'avance : c'est que nous en avons fait un objet.

Comme l'événement, la surprise contredit donc les conditions de l'expérience tout en devant d'une certaine façon s'inscrire dans l'expérience. Cette contradiction de l'expérience qui est encore une forme (paradoxale) d'expérience reçoit dans Étant donné le nom de contreexpérience. Nous en trouvons au $\S 22$ d'Étant donné trois exemples paradigmatiques, empruntés toutefois, non pas aux expériences de l'existence quotidienne, mais à certaines expériences emblématiques ayant reçu un traitement particulier dans la tradition philosophique. Ainsi, en premier lieu, l'épreuve de l'infini chez Descartes, sous la forme de la présence de l'idée d'infini en nous, qui exerce sur nous, précisément, "la surprise de l'admiration ${ }^{28}$. En deuxième position, nous trouvons l'exemple du sublime kantien qui, de façon encore plus prégnante, « porte les caractères ${ }^{29} \mathrm{du}$ phénomène saturé. L'expérience kantienne du sublime est en effet l'expérience de la démesure de la nature, de sa violence, voire de sa monstruosité qui nous écrase sans toutefois nous détruire. Le troisième exemple est sans doute plus étonnant: il s'agit de la conscience intime du temps selon Husserl, c'est-à-dire de l'expérience de la temporalité immanente que nous faisons chacun. Or s'il y a bien, comme l'analyse de cet exemple l'affirme, une imprévisibilité foncière du temps de la conscience, c'est que nous devons être, par définition, capables (ou passibles) de nous surprendre nous-mêmes, ce qui reviendrait à inscrire la surprise, non seulement dans l'expérience des choses du monde ou d'autrui, mais au cœur de l'expérience que chacun fait de lui-même.

Nous pouvons prolonger toute cette analyse du $\S 22$ avec des exemples plus banals: peut-on, par exemple, voir un arc-en-ciel sans être surpris ? Recevoir ou ouvrir un cadeau sans être surpris (même si nous risquons aussi - et c'est le risque même de toute expérience d'être déçus) ? Il faut en effet reconnaître, avec la «banalité de la saturation » (pour reprendre le titre du dernier chapitre de Le visible et le révélé (2005)), la banalité de la surprise, qui peut aller jusqu'à être organisée et planifiée (par exemple sous la forme d'une fête surprise). Il ne faut donc peut-être plus penser la surprise comme exception et anomalie, mais s'interroger sur le sens de sa potentielle ubiquité. Nous invoquerons en ce sens un extrait du deuxième chapitre de $D e$

${ }^{28}$ Ibid., p. 305.

${ }^{29}$ Ibid., p. 306. 
surcroît (2001), chapitre intitulé «L'événement ou le phénomène advenant » et qui prolonge les analyses d'Étant donné :
Même cette salle apparaît, en effet, sur le mode de l'événement. $\mathrm{Qu}$ 'on n'objecte pas qu'elle s'offre à voir comme un objet: quatre murs, un [...] plafond [...], un certain nombre de sièges, disponibles comme autant d'étants permanents et subsistants, et qui demeurent, attendant que nous les habitions en les utilisant ou que nous en constations la substance. Mais cette permanence en attente signifie ici curieusement le contraire de la disponibilité objective. [...] Car, en tant que toujours là, disponible à notre entrée et à notre usage, cette salle s'impose à nous comme préalable à nous, étant sans nous, quoique pour nous, qui donc surgit à notre vue comme un fait inattendu, imprévisible, venant d'un passé incontrôlable. Cette sur- prise ne surgit pas seulement aux salles de tel palais romain, souvent longé lors des promenades extérieures de touriste ignorant ou des marches pressées d'un habitant de la Ville éternelle, mais dont, parfois exceptionnellement invités à y pénétrer, nous découvrons d'un coup la splendeur imprévisible et restée jusqu'alors invue. Cette surprise se déclenche en fait aussi bien pour la Salle des Actes - déjà là, surgie d'un passé que nous ignorons, [...] chargée d'une histoire excédant la mémoire [...], elle s'impose à moi en apparaissant ; j'y entre moins qu'elle ne m'advient elle-même, m'englobe et m'en impose. Ce « déjà » atteste l'événement ${ }^{30}$.

La phénoménologie de la saturation, comme phénoménologie de la surprise et de l'événement, n'est donc pas une phénoménologie de l'extraordinaire détachée de la quotidienneté : en affirmant la banalité de la saturation, il s'agit au contraire d'inscrire l'extraordinaire à même la quotidienneté et de montrer que la surprise qualifie le surgissement même du phénomène, le mouvement même de la phénoménalisation. Plutôt qu'une exception ou une expérience limite, la surprise pourrait ainsi désigner une condition: celle, pourrait-on dire en citant Étant donné, d'être «essentiellement surpris par l'événement plus originel qui [m]e déprend de $[\mathrm{m}]$ oi ${ }^{31}$, événement qui n'est pas nécessairement traumatisme mais en premier lieu rencontre : il y a apparaître.

Nous arrivons à la conclusion de cette analyse dont l'objectif a été de montrer la richesse des enjeux que recèle la reprise du thème de la surprise à l'intérieur de la phénoménologie de la donation et de la saturation. Nous avons voulu suggérer tout d'abord que la saturation, pensée comme surcroît et excédent, permet de proposer une compréhension proprement positive de la surprise, qui ne se réduit

${ }^{30}$ J.-L. Marion, De surcroît. Études sur les phénomènes saturés, Paris, PUF 2001, p. 37-38.
${ }^{31}$ ED, p. 303. Nous soulignons. 
plus à l'effet d'une défaillance comme l'inattention (Heidegger) ou au déguisement d'une déception (Husserl). Mais ce faisant, nous avons tenté également de montrer que, tout comme elle s'en trouve éclairée, la surprise peut elle-même éclairer la pensée de la saturation. Nous avons donc tâché d'élargir la portée descriptive de la surprise pour en faire une détermination, non plus seulement de l'appel qui institue l'interloqué ou l'adonné, mais du phénomène saturé lui-même. L'excédent ou le surcroît qui constitue la marque de la saturation peut en effet être déterminé comme surprise, et ce pour préciser à la fois la manière dont il advient et la manière dont il est reçu. Plus que l'événement, la surprise nous semble à même de permettre de tenir ensemble ces deux versants : la saturation et l'épreuve qu'on en fait, ou l'advenir du phénomène saturé et la manière dont il est reçu. C'est en effet, en dernière instance, la possibilité d'être surpris (pour reprendre l'expression de Heidegger) qui décide de la différence entre le sujet métaphysique ou transcendantal et l'adonné.

Mais la double contradiction de la subjectivité et des conditions de l'expérience ne doit pas être comprise ici comme négation : plus qu'à une logique de la négation, elle obéit à une logique de l'excédent. C'est à la surprise qu'il revient d'indiquer, avec une plus grande clarté, la manière dont l'excédent de l'expérience se fait surcroît de la saturation. Dans la surprise se donnent ainsi ensemble l'avènement de la saturation et l'avènement de l'adonné et s'atteste leur co-appartenance. Si l'on peut donc affirmer, comme nous l'avons avancé au début de notre propos à titre d'hypothèse, que le phénomène en tant que donné est une surprise par la saturation, une dernière application de cette thèse serait de reformuler la différence entre objet et événement (entre ces deux régimes de phénoménalités qu'Étant donné distinguait comme phénomènes de droit commun et phéno-mènes saturés), que propose le $\S 27$ de Certitudes négatives ${ }^{32}$, en différence entre ce qui se donne sans surprise et ce qui se donne par surprise.

32 J.-L. Marion, Certitudes négatives, Paris, Grasset, 2010. 